\title{
Optimal exercise of an executive stock option by an insider
}

\author{
Michael Monoyios* \\ Mathematical Institute \\ University of Oxford
}

\author{
ANDREW NG \\ Mathematical Institute \\ University of Oxford
}

August 16, 2010

\begin{abstract}
We consider an optimal stopping problem arising in connection with the exercise of an executive stock option by an agent with inside information. The agent is assumed to have noisy information on the terminal value of the stock, does not trade the stock or outside securities, and maximises the expected discounted payoff over all stopping times with regard to an enlarged filtration which includes the inside information. This leads to a stopping problem governed by a time-inhomogeneous diffusion and a call-type reward. We establish conditions under which the option value exhibits time decay, and derive the smooth fit condition for the solution to the free boundary problem governing the maximum expected reward, and derive the early exercise decomposition of the value function. The resulting integral equation for the unknown exercise boundary is solved numerically and this shows that the insider may exercise the option before maturity, in situations when an agent without the privileged information may not. Hence we show that early exercise may arise due to the agent having inside information on the future stock price.
\end{abstract}

\section{Introduction}

In this paper we model the exercise decision of an insider who is granted an executive stock option (ESO). The agent is an employee who is granted a single American-style option on a stock of his employing company. This executive is barred from trading the stock, does not trade other securities either, and has some inside information on the future evolution of the stock at the terminal date of the option. The executive is modelled as risk-neutral, so has a linear utility function, and hence maximises the discounted expectation under the physical measure $P$ of the option payoff at the exercise time. We do not endow the agent with risk-averse preferences as we wish to focus exclusively on the role of inside information on the exercise decision, and this is also the reason for assuming away all other trading opportunities, as well as other contractual complications that are common in ESOs, such as a vesting period, resetting of strikes, partial exercise opportunities, job termination, and so on.

The exercise time is a stopping time with respect to an enlarged filtration formed by augmenting a filtration $\mathbb{F}$ with the $\sigma$-algebra of a random variable $L$, which corresponds to noisy information on the value of the stock at the option maturity time $T$. Writing the stock dynamics under the enlarged filtration $\mathbb{F}^{L}$, the stock price is governed by a time-inhomogeneous diffusion with state and time-dependent drift and constant volatility, and we are faced with an optimal stopping problem governed by the time-inhomogeneous diffusion. The enlargement of filtration, leading to a state and time-dependent drift, leads to the theoretical possibility of early exercise. We establish that early exercise can occur and provide a numerical computation of the early exercise boundary.

We establish the equivalence between the optimal stopping problem and a free boundary PDE. We further establish that the value function governing the maximum expected reward

\footnotetext{
*Corresponding author. We thank Peter Bank, Kasper Larsen, Goran Peskir, Martin Schweizer, Mihalis Zervos, two anonymous referees and participants at the Workshop on Foundations of Mathematical Finance (Fields Institute, 2010) for helpful suggestions.
} 
exhibits time decay for suitably low realisations of $L$, and for zero interest rate, regardless of $L$. In these cases we prove that the value function satisfies the smooth-fit condition at the free boundary, and from this we derive the early exercise decomposition of the value function into a European-style payoff and an early exercise premium. This leads to an integral equation characterising the free boundary, which is solved numerically. The numerical results show that the insider can indeed exercise the ESO prior to maturity in situations in which an executive without the inside information would not. Hence, we show that privileged information can also be a factor contributing to the early exercise of ESOs.

The rest of the paper is as follows. Section 2 describes the model and the optimal stopping problems for an insider and a non-insider. Section 3 contains our main results. We analyse the value function of the insider's discounted ESO value as a function of log-stock price. We use ideas of stochastic flows to establish convexity and monotonicity of the value function, derive the free boundary PDE, conditions under which the ESO value exhibits time decay, and the smooth fit condition at the exercise boundary. We use these results to derive an early exercise decomposition for the ESO value, and a resulting integral equation for the exercise boundary. The properties are well-known in standard American option problems with constant parameters under a martingale measure, but our problem is rather non-standard, containing a time-inhomogeneous drift term not equal to the interest rate, since valuation is performed under the physical measure. We solve the integral equation numerically and present results which show that the insider can be induced to early exercise by possessing privileged information. Section 4 concludes.

\section{The model}

We have a single stock price process $S=\left(S_{t}\right)_{0 \leq t \leq T}$ defined on a complete probability space $(\Omega, \mathcal{F}, P)$ equipped with a filtration $\mathbb{F}=\left(\mathcal{F}_{t}\right)_{0 \leq t \leq T}$ that satisfies the usual conditions of rightcontinuity and completeness. A $(P, \mathbb{F})$-Brownian motion $B=\left(B_{t}\right)_{0 \leq t \leq T}$ drives the stock price, which follows the geometric Brownian motion

$$
d S_{t}=\mu S_{t} d t+\sigma S_{t} d B_{t}
$$

where $\mu$ and $\sigma>0$ are known constants. There is a constant risk-free interest rate $r \geq 0$.

It will be sometimes be more convenient to work with the $\log$-stock price $X:=\log S$, satisfying

$$
X_{t}=X_{0}+\gamma t+\sigma B_{t}, \quad 0 \leq t \leq T
$$

where $\gamma:=\mu-\sigma^{2} / 2$.

Our financial agent (an executive) is an employee of the firm whose stock price is $S$, and is awarded at time zero a single American-style call option on the stock with maturity $T$. We assume that the agent is barred from trading $S$ or outside securities, that there is no opportunity for partial exercise of the option, and we take the agent's preferences to be risk-neutral, so he maximises the expected discounted payoff under the objective probability measure $P$. Grasselli and Henderson [6] or Rogers and Scheinkman [16] focus on the effects of risk aversion and outside trading on early and block exercise. We do not introduce contractual complications that sometimes feature in ESOs, as done by Sircar and co-authors [12, 13, 18]. We exclude the above features of ESOs in order to focus exclusively on the impact of inside information on the agent's optimal stopping problem of when to exercise the option. In particular, we can examine a case in which the absence of inside information results in no early exercise, and we can then show that the introduction of additional information can lead to early exercise, and we compute the early exercise boundary numerically. Even with the simplifications that we make, we shall see that we are nevertheless faced with a non-standard American problem with a timeinhomogeneous diffusion for the stock, so that many standard properties of the value function are not known a priori and have to be established. These properties include monotonicity and convexity in the log-stock price, time decay, and the smooth fit condition (continuity of the first spatial derivative) at the optimal exercise boundary. 
The agent has inside knowledge at time zero of an $\mathcal{F}$-measurable random variable $L$, corresponding to noisy knowledge of the terminal log-stock price $X_{T}$. We shall sometimes refer to this agent as the insider or the executive. We shall also consider an agent whose information is represented by the filtration $\mathbb{F}$, so who does not have the privileged information. This agent will sometimes be referred to as the regular agent or as the non-executive.

The random variable $L$ will be given by

$$
L=a X_{T}+(1-a) \epsilon, \quad 0<a<1,
$$

where $\epsilon$ is a standard normal random variable independent of $\mathbb{F}$. Hence, the executive's information flow is represented by the enlarged filtration $\mathbb{F}^{L}=\left(\mathcal{F}_{t}^{L}\right)_{0 \leq t \leq T}$, defined by

$$
\mathcal{F}_{t}^{L}:=\mathcal{F}_{t} \vee \sigma(L), \quad 0 \leq t \leq T .
$$

See Danilova, Monoyios and Ng [1] for similar examples of such inside information within the context of partial information investment problems. The dynamics of the log-stock price with respect to the enlarged filtration are given by classical enlargement of filtration methods (see Yor [19]) in the following lemma.

Lemma 1. With respect to the enlarged filtration $\mathbb{F}^{L}$, the dynamics of the log-stock price are

$$
d X_{t}=\frac{1}{T_{a}-t}\left(\gamma\left(T_{a}-T\right)+\frac{L}{a}-X_{t}\right) d t+\sigma d B_{t}^{L}
$$

where $B^{L}$ is an $\mathbb{F}^{L}$-Brownian motion and $T_{a}$ is the modulated time defined by

$$
T_{a}:=T+\left(\frac{1-a}{a \sigma}\right)^{2}, \quad 0<a<1
$$

Proof. Classical enlargement of filtration results (Theorem 12.1 in Yor [19]) imply that the $\mathbb{F}$-Brownian $B$ has a semi-martingale decomposition with respect to $\mathbb{F}^{L}$ of the form

$$
B_{t}=B_{t}^{L}+\int_{0}^{t} \nu(L, u) d u, \quad 0 \leq t \leq T
$$

with $B^{L}$ an $\mathbb{F}^{L}$-Brownian motion, and the process $(\nu(L, t))_{0 \leq t \leq T}$, is called the information drift, given by the logarithmic derivative of the conditional density of $L$ given $\mathcal{F}_{t}$. This results in

$$
B_{t}=B_{t}^{L}+\int_{0}^{t} \frac{L-a\left(X_{0}+\gamma T+\sigma B_{u}\right)}{a \sigma\left(T_{a}-u\right)} d u, \quad 0 \leq t \leq T,
$$

and combining this with (1), the lemma follows.

\subsection{The optimal stopping problems}

Denote by $\mathcal{T}$ the set of all stopping times with respect to the filtration $\mathbb{F}$, and by $\mathcal{T}^{L}$ the set of all stopping times with respect to the enlarged filtration $\mathbb{F}^{L}$. Introduce the following subsets of $\mathcal{T}$ and $\mathcal{T}^{L}$ :

$$
\begin{aligned}
& \mathcal{T}_{t, T}:=\{\tau \in \mathcal{T} \mid P(\tau \in[t, T])=1\}, \quad 0 \leq t \leq T<\infty \\
& \mathcal{T}_{t, T}^{L}:=\quad\left\{\tau \in \mathcal{T}^{L} \mid P(\tau \in[t, T])=1\right\}, \quad 0 \leq t \leq T<\infty
\end{aligned}
$$

Of course, we have $\mathcal{T}_{0, T} \equiv \mathcal{T}$ and $\mathcal{T}_{0, T}^{L} \equiv \mathcal{T}^{L}$.

The executive stock option is an American call with strike $K \geq 0$. If exercised at time $\tau \in[0, T]$, the discounted payoff at time zero is $Y_{\tau}$, where $Y=\left(Y_{t}\right)_{0 \leq t \leq T}$ is the reward process, defined by

$$
Y_{t}:=e^{-r t}\left(e^{X_{t}}-K\right)^{+}, \quad 0 \leq t \leq T
$$


assumed to satisfy

$$
E\left[\sup _{0 \leq t \leq} Y_{t}\right]<\infty .
$$

The insider's (that is, the executive's) optimal stopping problem is to find a stopping time $\tau^{*} \in \mathcal{T}^{L}$ to achieve the maximal expected reward

$$
V_{0}(L):=\sup _{\tau \in \mathcal{T}^{L}} E\left[Y_{\tau} \mid \mathcal{F}_{0}^{L}\right] .
$$

Notice that the supremum is over stopping times of the enlarged filtration $\mathbb{F}^{L}$, so we emphasise this with the conditioning on the enlarged initial $\sigma$-field $\mathcal{F}_{0}^{L}$. The maximal expected reward $V_{0}(L)$ is thus an $\mathcal{F}_{0}^{L}$-measurable random variable (though from the perspective of the insider, $L$ is a known constant) and the relevant dynamics of the state variable $X$ are given by Lemma 1. When no confusion arises we suppress the dependence on $L$ of $V_{0}$.

The non-executive faces a similar optimal stopping problem, but over $\mathbb{F}$-stopping times, so in this case $X$ is given by (1). We denote the non-executive's maximal expected reward at time zero by

$$
V_{0}^{0}:=\sup _{\tau \in \mathcal{T}} E\left[Y_{\tau}\right]
$$

where the expectation is conditional upon the (assumed trivial) $\sigma$-algebra $\mathcal{F}_{0}$.

\subsection{Benchmark case: $\mu \geq r$ and no inside information}

For $\mu \geq r$, the reward process $Y$ is a $(P, \mathbb{F})$-submartingale, so the regular agent's value for the American ESO coincides with the European value: $V_{0}^{0}=E\left[Y_{T}\right]$. In particular, the exercise time $\tau=T$ is optimal for the regular agent. This slightly artificial conclusion derives from the fact that there are no trading opportunities for the regular agent and also that the agent has a linear utility function. This result serves as a useful benchmark for us. Given the same trading opportunities (none) and the same preferences for the insider as the regular agent, our main goal is to show that inside information on the stock can result in early exercise, because the drift of the stock becomes time and price-dependent.

\subsection{The insider's problem}

In this section we analyse the optimal stopping problem for the insider. The log-stock price follows the time-inhomogeneous diffusion of Lemma 1, which we write as

$$
d X_{t}=\beta\left(t, X_{t}\right) d t+\sigma d B_{t}^{L},
$$

where $\beta(t, x) \equiv \beta(t, x ; L)$ is given by

$$
\beta(t, x)=\frac{C-x}{T_{a}-t}, \quad C:=\gamma\left(T_{a}-T\right)+\frac{L}{a} .
$$

Given an initial condition $X_{t}=x \in \mathbb{R}$, for $t \in[0, T]$, the solution to (5) is the Gaussian process $\left(X_{u}\right)_{t \leq u \leq T}$ given by

$$
X_{u}=x+\beta(t, x)(u-t)+\sigma\left(T_{a}-u\right) \int_{t}^{u} \frac{1}{T_{a}-\rho} d B_{\rho}^{L}, \quad 0 \leq t \leq u \leq T .
$$

In particular, the transition density $p(t, x ; u, y)$ for moving from $X_{t}=x$ to $X_{u} \in d y$ at $u \geq t$ is given explicitly by

$$
p(t, x ; u, y)=\frac{1}{\Sigma(t ; u) \sqrt{2 \pi}} \exp \left[-\frac{1}{2}\left(\frac{y-m(t, x ; u)}{\Sigma(t ; u)}\right)^{2}\right], \quad x, y \in \mathbb{R}, \quad 0 \leq t \leq u \leq T,
$$

where $m(t, x ; u)$ and $\Sigma(t ; u)>0$ are given by

$$
m(t, x ; u)=x+\beta(t, x)(u-t), \quad \Sigma^{2}(t ; u)=\sigma^{2}\left(\frac{T_{a}-u}{T_{a}-t}\right)(u-t) .
$$


For a starting time $t \in[0, T]$ the maximal expected discounted payoff is given by the $\mathbb{F}^{L}$ adapted process

$$
V_{t} \equiv V_{t}(L):=\operatorname{ess~sup}_{\tau \in \mathcal{T}_{t, T}^{L}} E\left[e^{-r(\tau-t)}\left(e^{X_{\tau}}-K\right)^{+} \mid \mathcal{F}_{t}^{L}\right]=e^{r t} \underset{\tau \in \mathcal{T}_{t, T}^{L}}{\operatorname{ess} \sup } E\left[Y_{\tau} \mid \mathcal{F}_{t}^{L}\right], \quad 0 \leq t \leq T .
$$

We are thus led to consider the $\mathbb{F}^{L}$-adapted process $U$ defined by

$$
U_{t} \equiv U_{t}(L):=\operatorname{ess} \sup _{\tau \in \mathcal{T}_{t, T}^{L}} E\left[Y_{\tau} \mid \mathcal{F}_{t}^{L}\right], \quad 0 \leq t \leq T
$$

satisfying $U_{t}=e^{-r t} V_{t}$ a.s., for any $t \in[0, T]$.

Classical optimal stopping theory (Appendix D of Karatzas and Shreve [11]) characterises the solution to the optimal stopping problem (10) as follows. There exists a non-negative càdlàg $\left(P, \mathbb{F}^{L}\right)$-supermartingale $U=\left(U_{t}\right)_{0 \leq t \leq T}$, the Snell envelope of $Y$, such that $U$ is the smallest $\left(P, \mathbb{F}^{L}\right)$-supermartingale that dominates $Y$, with $U_{T}=Y_{T}$ a.s. A stopping time $\tau^{*} \in \mathcal{T}^{L}$ is optimal for the problem (10) starting at time zero if and only if $U_{\tau^{*}}=Y_{\tau^{*}}$ a.s., and the stopped supermartingale $U^{\tau^{*}}$ defined by $U_{t}^{\tau^{*}}:=U_{\tau^{*}} \wedge t, 0 \leq t \leq T$, is a $\left(P, \mathbb{F}^{L}\right)$-martingale. The smallest optimal stopping time in $\mathcal{T}_{t, T}^{L}$ for the problem (10) is $\tau_{t}^{*}$, defined by

$$
\tau_{t}^{*}:=\inf \left\{\rho \in[t, T] \mid U_{\rho}=Y_{\rho}\right\} \wedge T, \quad 0 \leq t \leq T .
$$

\section{The value function}

We are interested in the optimal stopping problem with reward process

$$
Y_{t}=e^{-r t}\left(e^{X_{t}}-K\right)^{+}=: f\left(t, X_{t}\right),
$$

where $f:[0, T] \times \mathbb{R} \rightarrow \mathbb{R}^{+}$is the continuous non-negative function given by

$$
f(t, x):=e^{-r t}\left(e^{x}-K\right)^{+}
$$

and where $\left(X_{u}\right)_{t \leq u \leq T}$ is the solution (7) of (5) with $X_{t}=x$ deterministic, for $t \in[0, T]$. For a fixed value of the random variable $L$, say $L=\ell \in \mathbb{R}$, we define the value function $F:[0, T] \times \mathbb{R} \rightarrow \mathbb{R}^{+}$by

$$
F(t, x) \equiv F(t, x ; \ell):=\sup _{\tau \in \mathcal{T}_{t, T}^{L}} E\left[f\left(\tau, X_{\tau}\right) \mid X_{t}=x, L=\ell\right] .
$$

Then, in a very general continuous-time Markov setting, $F$ is a continuous function and the process $U=\left(F\left(t, X_{t}\right)\right)_{0 \leq t \leq T}$ is the Snell envelope of $Y=\left(f\left(t, X_{t}\right)\right)_{0 \leq t \leq T}$ (see for instance El Karoui, Lepeltier and Millet [5]).

The insider's value process for the ESO is $\left(V\left(t, S_{t} ; L\right)\right)_{0 \leq t \leq T}$, where $V:[0, T] \times \mathbb{R}^{+} \rightarrow \mathbb{R}^{+}$ is given by

$$
V(t, s) \equiv V(t, s ; \ell):=\sup _{\tau \in \mathcal{T}_{t, T}^{L}} E\left[e^{-r(\tau-t)}\left(S_{\tau}-K\right)^{+} \mid S_{t}=s, L=\ell\right],
$$

and we suppress dependence on $L$ when no confusion arises. Hence the value functions $F$ and $V$ are related according to

$$
e^{-r t} V(t, s(x))=F(t, x), \quad \text { with } s(x):=e^{x} .
$$

The (smallest) optimal stopping time for the problem (11) starting at time $t \in[0, T]$ with $X_{t}=x$ is $\tau^{*}(t, x)$ given by

$$
\tau^{*}(t, x)=\inf \left\{\rho \in[t, T] \mid F\left(\rho, X_{\rho}\right)=f\left(\rho, X_{\rho}\right)\right\}=\inf \left\{\rho \in[t, T] \mid V\left(\rho, S_{\rho}\right)=\left(S_{\rho}-K\right)^{+}\right\} .
$$

The continuation region $\mathcal{C}$ is defined by

$$
\mathcal{C}:=\{(t, x) \in[0, T) \times \mathbb{R} \mid F(t, x)>f(t, x)\}=\left\{(t, s) \in[0, T) \times \mathbb{R}^{+} \mid V(t, s)>(s-K)^{+}\right\} .
$$


Since $F, V$ are continuous, $\mathcal{C}$ is open. This suggests (and we show below) that there is function $x^{*}:[0, T] \rightarrow \mathbb{R}$ (respectively, $s^{*}:[0, T] \rightarrow \mathbb{R}^{+}$), the critical log-stock price (respectively, critical stock price) or optimal early exercise boundary, such that the option is exercised the first time the $\log$-stock price exceeds $x^{*}(t)$. Since it is never optimal to exercise if the stock is below the strike $K$, we must have $x^{*}(t) \geq \log K$ for all $t \in[0, T]$.

We shall characterise the early exercise boundary and the value function $F$ as a solution to a free boundary problem, and we also establish the smooth fit condition at the boundary that is common in many optimal stopping problems. This is not guaranteed and in general needs to be verified on a case by case basis. This is the situation we are faced with here, as we are dealing with a non-standard American option problem involving a stock with a state and time-dependent drift.

\subsection{Convexity and monotonicity of the value function in $x$}

We wish to show that the value function $F$ is increasing and convex in $x$. Although these properties do not necessarily imply similar properties for the ESO value $V$ in the stock price, they will be sufficient to allow us to characterise the exercise boundary and establish bounds on the derivative $F_{x}$, which are ingredients we need to obtain the free boundary PDE and the smooth fit condition satisfied by $F$. These then lead easily to a corresponding free boundary $\mathrm{PDE}$ and smooth fit condition for $V$.

We shall utilise ideas of stochastic flows applied to the log-stock price. We write $X(x)$ for the log-stock price with initial condition $X_{0}=x$, considered as the solution to a diffusion SDE with time and state-dependent drift. In Lemma 2 we show that the map $x \rightarrow X(x)$ is non-decreasing, and give a condition on the drift $\beta$ of $X$ for this map to be convex in $x$. This condition is indeed satisfied in our specific model. From the properties of $x \rightarrow X(x)$ we deduce the corresponding properties for the map $x \rightarrow F(\cdot, x)$.

Note that for a diffusion with time and state dependent drift, properties such as monotonicity and convexity in the initial condition are not automatic, so the obvious properties of the map $x \rightarrow X(x)$ under $\mathbb{F}$ do indeed need to be shown to hold under $\mathbb{F}^{L}$.

An alternative to our approach would be to use a technique due to El Karoui et al [4]. They prove convexity of standard American option prices with respect to stock price (so evaluated under a martingale measure) in diffusion models with deterministic interest rate. They also employ ideas of stochastic flows, first to show the property for European prices, then, adapting an iterative procedure found in El Karoui [3], they extend the result to American prices. This approach can be shown to work in our model, since the European option value can be written as an integral with respect to the transition density of $X$, given in (8). ${ }^{1}$ Indeed, we adapt this technique later for part of our analysis of the time decay property of the American ESO value: see the proof of Theorem 3.

In principle one might try to use our techniques to prove convexity and monotonicity of ESO value function $V$ in the starting stock price $S_{t}=s$, for any $t \in[0, T]$. This does not appear to be straightforward using our methods, because it does not appear easy to prove that the map $s \rightarrow S(s)$ is increasing and convex for a general diffusion. Indeed, we shall see in Remark 1 that, when we use the explicit solution (7) for $X(x)$, the map $x \rightarrow X(x)$ is indeed increasing and convex, but that the map $s \rightarrow S(s)$ is increasing but not convex.

It is well known that convexity of American option prices with respect to stock price does not immediately follow from the convexity of the payoff process when the return distribution of the stock depends on the stock price, as shown by Merton [14] (Theorem 10 and the counter-example in Appendix 1). Other authors have also analysed convexity of American option values with respect to stock price. Ekstrom [2] used stochastic time changes and a limiting argument based on approximating American option by a Bermudan option, and Hobson [7] utilised coupling methods. Similar to [4], these papers consider standard American pricing problems under a risk-neutral measure, with a deterministic rate of interest. We have a rather non-standard problem where the stock price drift is not the interest rate, and in addition is both state and

\footnotetext{
${ }^{1}$ We thank an anonymous referee for pointing this out.
} 
time-dependent. For these reasons, we cannot directly read off the required properties of the value function from these papers.

For simplicity consider a starting time $t=0$. The same ideas apply to any starting time $t \in[0, T]$. Consider the log-stock price with initial condition $X_{0}=x$, and write $X \equiv X(x)$, following

$$
X_{t}(x)=x+\int_{0}^{t} \beta\left(u, X_{u}(x)\right) d u+\sigma B_{t}^{L}, \quad 0 \leq t \leq T .
$$

We may choose versions of $\left(X_{t}(x)\right)_{0 \leq t \leq T}$ which for each $t \in[0, T]$ and each $\omega \in \Omega$ are diffeomorphisms in $x$ from $\mathbb{R} \rightarrow \mathbb{R}$. That is, the map $x \rightarrow X(x)$ is smooth.

Define

$$
b(t, x):=\frac{\partial}{\partial x} \beta(t, x), \quad D_{t}(x):=\frac{\partial}{\partial x} X_{t}(x) .
$$

Lemma 2. The map $x \rightarrow X(x)$ is increasing, and if $\beta_{x x}(t, y) \geq 0$, also convex.

Proof. We have

$$
D_{t}(x)=\exp \left(\int_{0}^{t} b\left(u, X_{u}(x)\right) d u\right)>0
$$

so $x \rightarrow X(x)$ is increasing.

Define $c(t, x):=b_{x}(t, x)=\beta_{x x}(t, x)$. Then

$$
\frac{\partial}{\partial x} D_{t}(x)=D_{t}(x) \int_{0}^{t} c\left(u, X_{u}(x)\right) D_{u}(x) d u,
$$

which is non-negative if $c(t, x) \geq 0$ for all $(t, x) \in[0, T] \times \mathbb{R}$. Then $x \rightarrow X(x)$ is convex.

Remark 1. Lemma 2 holds for a general diffusion with a time and state-dependent drift. Alternatively, with the explicit solution (7) we can directly compute

$$
D_{t}(x)=1-\frac{t}{T_{a}}>0, \quad \frac{\partial}{\partial x} D_{t}(x)=0,
$$

which directly shows that $x \rightarrow X(x)$ is increasing and convex. The same method applied to the map $s \rightarrow S(s)$ (the stock price with initial condition $S_{0}=s>0$ ) gives

$$
\frac{\partial}{\partial s} S_{t}(s)=\frac{S_{t}(s)}{s}\left(1-\frac{t}{T_{a}}\right)>0, \quad \frac{\partial^{2}}{\partial s^{2}} S_{t}(s)=-\frac{S_{t}(s)}{s^{2}}\left(1-\frac{t}{T_{a}}\right) \frac{t}{T_{a}}<0,
$$

so that $s \rightarrow S(s)$ is increasing, but not convex (though this does not necessarily imply that $s \rightarrow V(\cdot, s)$ is not convex).

Theorem 1. The map $x \rightarrow F(t, x)$ is increasing for any $t \in[0, T]$. Suppose $\beta_{x x}(t, x) \geq 0$. Then the map $x \rightarrow F(t, x)$ is convex for any $t \in[0, T]$.

Proof. We set $t=0$ without loss of generality. Then $F(x) \equiv F(0, x)$ is given by

$$
F(x)=\sup _{\tau \in \mathcal{T}^{L}} E\left[e^{-r \tau}\left(\exp \left(X_{\tau}(x)\right)-K\right)^{+} \mid \mathcal{F}_{0}^{L}\right]
$$

and where $X_{0}(x)=x$. Let $\tau^{*}(x) \in \mathcal{T}^{L}$ denote the optimal stopping time for the problem in (14). Then we may write

$$
F(x)=E\left[e^{-r \tau^{*}(x)}\left(\exp \left(X_{\tau^{*}(x)}(x)\right)-K\right)^{+}\right],
$$

where for brevity we have suppressed the conditioning on $\mathcal{F}_{0}^{L}$.

Since the map $x \rightarrow X(x)$ is increasing, we have, for $x_{0}<x_{1}$,

$$
\left(\exp \left(X_{\tau^{*}\left(x_{0}\right)}\left(x_{0}\right)\right)-K\right)^{+}<\left(\exp \left(X_{\tau^{*}\left(x_{0}\right)}\left(x_{1}\right)\right)-K\right)^{+} .
$$


Multiply both sides by $e^{-r \tau^{*}\left(x_{0}\right)}$, take expectations, and use the fact that $\tau^{*}\left(x_{0}\right)$ is sub-optimal for the starting state $x_{1}$, to obtain

$$
F\left(x_{0}\right)<E\left[e^{-r \tau^{*}\left(x_{0}\right)}\left(\exp \left(X_{\tau^{*}\left(x_{0}\right)}\left(x_{1}\right)\right)-K\right)^{+}\right] \leq F\left(x_{1}\right)
$$

which shows that $x \rightarrow F(x)$ is non-decreasing.

To establish convexity, define $x_{\lambda}:=\lambda x_{0}+(1-\lambda) x_{1}$ for $x_{0}<x_{1}$ and $\lambda \in[0,1]$. Using the property that $x \rightarrow X(x)$ is convex, we have that $x \rightarrow(\exp (X(x))-K)^{+}$is also convex. Hence

$$
\left(\exp \left(X_{\tau^{*}\left(x_{\lambda}\right)}\left(x_{\lambda}\right)\right)-K\right)^{+} \leq \lambda\left(\exp \left(X_{\tau^{*}\left(x_{\lambda}\right)}\left(x_{0}\right)\right)-K\right)^{+}+(1-\lambda)\left(\exp \left(X_{\tau^{*}\left(x_{\lambda}\right)}\left(x_{1}\right)\right)-K\right)^{+} .
$$

Multiplying by $\exp \left(-r \tau^{*}\left(x_{\lambda}\right)\right)$, taking expectations and using the fact that $\tau^{*}\left(x_{\lambda}\right)$ is sub-optimal for the starting states $x_{i}, i=0,1$, we obtain

$$
F\left(x_{\lambda}\right) \leq \lambda F\left(x_{0}\right)+(1-\lambda) F\left(x_{1}\right),
$$

so $x \rightarrow F(x)$ is convex.

\subsection{Free boundary problem for the value function}

As $F$ is increasing and convex, the exercise boundary $x^{*}(t)$ divides the domain of $F$ into the continuation region $\mathcal{C}$ and the stopping region $\mathcal{S}$, given by

$$
\mathcal{C}=\left\{(t, x) \in[0, T) \times \mathbb{R} \mid x<\log x^{*}(t)\right\}=\left\{(t, s) \in[0, T) \times \mathbb{R}^{+} \mid s<s^{*}(t)\right\},
$$

with $\mathcal{S}=\mathcal{C}^{c}$

Define the extended generator $\mathcal{L}$ of $X$ by

$$
\mathcal{L} g(t, x):=g_{t}(t, x)+\beta(t, x) g_{x}(t, x)+\frac{1}{2} \sigma^{2} g_{x x}(t, x)
$$

Denote the closure of the continuation region by $\overline{\mathcal{C}}$.

Theorem 2. The value function $F$ in (11) solves, in $\overline{\mathcal{C}}$, the free boundary problem

$$
\begin{aligned}
& \mathcal{L} F(t, x)=0, \quad(t, x) \in \mathcal{C}, \\
& F(t, x) \quad>e^{-r t}\left(e^{x}-K\right), \quad(t, x) \in \mathcal{C}, \\
& F\left(t, x^{*}(t)\right)=e^{-r t}\left(e^{x^{*}(t)}-K\right), \quad 0 \leq t \leq T, \\
& F(T, x)=e^{-r T}\left(e^{x}-K\right)^{+}, \quad x \in \mathbb{R} \text {. }
\end{aligned}
$$

Proof. This is by standard methods (Theorem 2.7.7 in Karatzas and Shreve [11]).

\subsection{The exercise boundary is non-increasing}

We now analyse the time decay of the ESO value, that is, that the map $t \rightarrow V(t, s)$ is nonincreasing, for any $t \in[0, T]$ and $s \in \mathbb{R}^{+}$. This property will imply that the exercise boundary is a non-increasing function of time. Recall the $\mathcal{F}_{0}^{L}$-measurable random variable $C$ in $(6)$.

Theorem 3. 1. If $C \leq \log K$ then the map $t \rightarrow V(t, s)$ is non-increasing.

2. If the interest rate is zero, then $t \rightarrow V(t, s)$ is non-increasing for any value of $C$.

Time decay for American-style claims cannot be expected to hold in general when the price dynamics are governed by a time-inhomogeneous process, as pointed out by Ekstrom [2]. He describes a drastic counter-example, in which volatility can jump from zero to a positive value at some future time. Time decay is often taken for granted, as longer-dated options have all the exercise opportunities of shorter-dated claims, so holds in time-homogeneous models. For this reason, there seems to be very little analysis of this property in the literature. 
Theorem 3 states that the time decay property always holds for zero interest rate. The same holds for standard American pricing problems (under a martingale measure) in diffusion models (see [2]). Regardless of the interest rate, time decay for the ESO holds for suitably low realisations of the random variable $L$. Indeed, $C<\log K$ corresponds (modulo the noise in the inside information, governed by the parameter $a \in(0,1)$ ) to knowledge that the stock price will end up below the strike. With this knowledge, it is intuitively plausible that the insider would exercise the option early, knowing that it will end up out of the money, and this would make the ESO less valuable as time progresses.

We shall use this property below to establish that the exercise boundary is non-increasing, which is an ingredient in our subsequent proof of the smooth pasting condition. An alternative to our approach would be to use an iterative procedure due to Muthuraman [15], which seeks to solve American option problems using a sequence of problems each with known exercise boundary, and with successively better approximations to the true boundary. This would be a good topic for future research, and might be able to show that the smooth pasting condition holds. ${ }^{2}$ In particular, this would imply that in fact the exercise boundary is non-increasing and that the time decay property is valid.

Proof of Theorem 3. The dynamics of the stock price with respect to the enlarged filtration $\mathbb{F}^{L}$ are

$$
d S_{t}=S_{t}\left[\alpha\left(t, S_{t}\right) d t+\sigma d B_{t}^{L}\right], \quad \alpha(t, s)=\beta(t, \log s)+\frac{1}{2} \sigma^{2} .
$$

Using the Tanaka-Meyer formula (Jeanblanc et al [9], Chapter 4) applied to the semi-martingale $S$, we have

$$
\begin{aligned}
e^{-r u}\left(S_{u}-K\right)^{+} & =e^{-r t}\left(S_{t}-K\right)^{+}-r \int_{t}^{u} e^{-r \rho}\left(S_{\rho}-K\right)^{+} d \rho+\int_{t}^{u} e^{-r \rho} \mathbb{1}_{\left\{S_{\rho}>K\right\}} d S_{\rho} \\
& +\frac{1}{2} \int_{t}^{u} e^{-r \rho} d L_{\rho}^{K}(S), \quad 0 \leq t \leq u \leq T
\end{aligned}
$$

where $L^{K}(S)$ denotes the local time of $S$ at level $K$. We take expectation given $S_{t}=s$ (and of course, implicitly, given $L=\ell$, with this dependence suppressed). It is not hard to verify that the stochastic integral is a $\left(P, \mathbb{F}^{L}\right)$ martingale, and we obtain, on using the dynamics (15),

$$
\begin{aligned}
E\left[e^{-r u}\left(S_{u}-K\right)^{+} \mid S_{t}=s\right] & =e^{-r t}(s-K)^{+} \\
& +E\left[\int_{t}^{u} e^{-r \rho}\left[\left(\alpha\left(\rho, S_{\rho}\right)-r\right) S_{\rho}+r K\right] \mathbb{1}_{\left\{S_{\rho>K\}}\right.} d \rho \mid S_{t}=s\right] \\
& +\frac{1}{2} E\left[\int_{t}^{u} e^{-r \rho} d L_{\rho}^{K}(S) \mid S_{t}=s\right], \quad 0 \leq t \leq u \leq T .
\end{aligned}
$$

We proceed formally for the moment, and indicate further below how to make the following argument rigorous. The local time may be represented as

$$
L_{t}^{K}(S)=\int_{0}^{t} \delta\left(S_{\rho}-K\right) d\langle S\rangle_{\rho}, \quad 0 \leq t \leq T,
$$

where $\delta(\cdot)$ is the Dirac delta function. We shall give meaning to this heuristic expression further below. Using this representation of $L^{K}(S)$ we convert (16) into

$$
E\left[e^{-r(u-t)}\left(S_{u}-K\right)^{+} \mid S_{t}=s\right]-(s-K)^{+}=E\left[\int_{t}^{u} e^{-r(\rho-t)} \mathcal{A}\left(\rho, S_{\rho}\right) d \rho \mid S_{t}=s\right], \quad 0 \leq t \leq u \leq T,
$$

where

$$
\mathcal{A}(t, s):=[(\alpha(t, s)-r) s+r K] \mathbb{1}_{\{s>K\}}+\frac{1}{2} \sigma^{2} s^{s} \delta(s-K), \quad 0 \leq t \leq T, \quad s \in \mathbb{R}^{+} .
$$

\footnotetext{
${ }^{2}$ We thank an anonymous referee for pointing us towards this reference.
} 
Jacka and Lynn [8] use a similar construction to (17), but for smooth payoff functions, to analyse time decay of optimal stopping problems governed by diffusions.

Now consider two times $t_{0}, t$ satisfying $0 \leq t_{0}<t \leq T$. Suppose that $(t, s) \in \mathcal{C}$. Let $\tau^{*}(t, s)$ denote the optimal stopping time for starting state $(t, s)$ and define $v \geq 0$ by $\tau^{*}(t, s)=: t+v$. Applying (17) between $t$ and $t+v$, we obtain

$$
0<V(t, s)-(s-K)^{+}=E\left[\int_{t}^{t+v} e^{-r(\rho-t)} \mathcal{A}\left(\rho, S_{\rho}\right) d \rho \mid S_{t}=s\right] .
$$

Since $t_{0}+v$ is in general sub-optimal for the starting state $\left(t_{0}, s\right)$, the same argument applied over $\left[t_{0}, t_{0}+v\right]$ gives

$$
V\left(t_{0}, s\right)-(s-K)^{+} \geq E\left[\int_{t_{0}}^{t_{0}+v} e^{-r\left(\rho-t_{0}\right)} \mathcal{A}\left(\rho, S_{\rho}\right) d \rho \mid S_{t_{0}}=s\right] .
$$

From (18) and (19) we see that if $\mathcal{A}(t, s)$ is non-increasing in $t$, then we will have

$$
V\left(t_{0}, s\right)-(s-K)^{+} \geq V(t, s)-(s-K)^{+}>0,
$$

implying that value function is non-increasing in time.

The condition that $\mathcal{A}(t, s)$ is non-increasing in $t$ translates to

$$
\frac{(C-\log s)}{\left(T_{a}-t\right)^{2}} \mathbb{1}_{\{s>K\}} \leq 0 .
$$

This condition is clearly satisfied whenever $s \leq K$. When $s>K$ (which is the case whenever $(t, s) \in \mathcal{C})$ it will always be satisfied for $C \leq \log K$, and with the outline below of how to make the above arguments fully rigorous, this proves the first statement in the theorem.

To be fully rigorous, one must give precise meaning to the representation of the local time in terms of the Dirac delta function. This can be done in the classical manner in which the generalised Itô formula for convex functions is established, by approximating the Dirac delta function $\delta(x)$ by a sequence of probability densities with increasing concentration at the origin. This type of argument can be found in Sections 3.6 and 3.7 of Karatzas and Shreve [10] and is outlined below.

One defines a sequence of probability density functions (or mollifiers, positive $C^{\infty}$ functions with compact support that integrate to 1) $\left(\varphi_{n}(x)\right)_{n \in \mathbb{N}}$ as well as a sequence of functions $\left(u_{n}(x)\right)_{n \in \mathbb{N}}$, given by

$$
u_{n}(x):=\int_{-\infty}^{x} \int_{-\infty}^{y} \varphi_{n}(z-K) d z d y, \quad x \in \mathbb{R}, \quad n \geq 1,
$$

such that the following limiting relations hold:

$$
\lim _{n \rightarrow \infty} u_{n}(x)=(x-K)^{+}, \quad \lim _{n \rightarrow \infty} u_{n}^{\prime}(x)=\lim _{n \rightarrow \infty} \int_{-\infty}^{x} \varphi_{n}(z-K) d z=\mathbb{1}_{\{x>K\}},
$$

as well as

$$
\lim _{n \rightarrow \infty} \int_{\mathbb{R}} u_{n}^{\prime \prime}(x) g(x) d x=\lim _{n \rightarrow \infty} \int_{\mathbb{R}} \varphi_{n}(x-K) g(x) d x \int_{\mathbb{R}} \delta(x-K) g(x) d x=g(K),
$$

for any Borel function $g(\cdot)$. Thus, in the limit as $n \rightarrow \infty$, the function $\varphi_{n}(\cdot)$ takes on the same properties as the Dirac delta.

One now applies the same arguments that led to (17) with $u_{n}(x)$ in place of $(x-K)^{+}$, so we are able to use the Itô formula because the $u_{n}$ are $C^{2}$. This gives

$$
E\left[e^{-r(u-t)} u_{n}\left(S_{u}\right) \mid S_{t}=s\right]-u_{n}(s)=E\left[\int_{t}^{u} e^{-r(\rho-t)} \mathcal{A}_{n}\left(\rho, S_{\rho}\right) d \rho \mid S_{t}=s\right],
$$


where

$$
\mathcal{A}_{n}(t, s):=\alpha(t, s) s u_{n}^{\prime}(s)+\frac{1}{2} \sigma^{2} s^{s} u_{n}^{\prime \prime}(s)-r u_{n}(s), \quad 0 \leq t \leq T, \quad s \in \mathbb{R}^{+} .
$$

With this is place one looks for conditions such that $\mathcal{A}_{n}(t, s)$ is non-increasing in $t$, and finally takes the limit as $n \rightarrow \infty$, drawing the same conclusions as before.

To prove the second part of the theorem, we need to establish that when $C>\log K$, then time decay holds provided $r=0$, since we already know that time decay is valid for $C \leq \log K$, regardless of $r$. We do this by adapting a procedure found in El Karoui et al [4], first considering the time decay of a European ESO, and then invoking a variant of an iterative procedure originally due to El Karoui [3] which allows one to infer that the American ESO will inherit whatever time decay property holds for the European ESO.

The European ESO value for starting state $(t, s) \in[0, T] \times \mathbb{R}^{+}$and maturity $u \leq T$ is given by

$$
V^{E}(t, s ; u)=E\left[e^{-r(u-t)}\left(S_{u}-K\right)^{+} \mid S_{t}=s\right],
$$

where the dependence on a given value of $L$ is suppressed as usual. A straightforward computation using the transition density (8) gives

$$
V^{E}(t, s ; u)=e^{-r(u-t)}\left[e^{b(t, s ; u)} \Phi(z(t, s ; u))-K \Phi(z(t, s ; u)-\Sigma(t ; u))\right],
$$

where $\Phi(\cdot)$ is the standard cumulative normal distribution function and

$$
b(t, s ; u)=m(t, \log s ; u)+\frac{1}{2} \Sigma^{2}(t ; u), \quad z(t, s ; u)=\Sigma(t ; u)+\frac{m(t, \log s ; u)-\log K}{\Sigma(t ; u)},
$$

with $m, \Sigma$ defined in (9). Differentiation with respect to $t$ gives

$$
\frac{\partial V^{E}}{\partial t}(t, s ; u)=e^{-r(u-t)}\left[\left(r+\frac{\partial m}{\partial t}+\Sigma \frac{\partial \Sigma}{\partial t}\right) e^{b} \Phi(z)-r K \Phi(z-\Sigma)+K \frac{\partial \Sigma}{\partial t} \Phi^{\prime}(z-\Sigma)\right],
$$

where we have suppressed arguments of functions for brevity. Since $\Phi$ and $\Phi^{\prime}$ are positive and $\partial \Sigma / \partial t$ is negative, the last two terms on the right hand side are negative, so the European ESO value will be guaranteed to be non-increasing with time provided that

$$
r+\frac{\partial m}{\partial t}(t, \log s ; u)+\Sigma(t ; u) \frac{\partial \Sigma}{\partial t}(t ; u) \leq 0
$$

This condition translates to

$$
C-\log s \geq r \frac{\left(T_{a}-t\right)^{2}}{T_{a}-u}-\frac{1}{2} \sigma^{2}\left(T_{a}-u\right) .
$$

Suppose $r=0$. We are ultimately interested in when the American ESO value will exhibit time decay, and since $V(t, s)=0$ for $s \leq K$, we only consider the case when $s>K$. Then, for the European ESO value to exhibit time decay in the region $s>K$ we require

$$
C-\log s \geq-\frac{1}{2} \sigma^{2}\left(T_{a}-u\right), \quad \text { when } s>K .
$$

Since the right hand side is negative, the condition will be guaranteed if $C \geq \log K$. Hence we conclude that for $r=0$ and $s>K, \partial V^{E} / \partial t(t, s ; u) \leq 0$ if $C \geq \log K$.

To complete the proof we now invoke the iterative procedure of El Karoui et al [4] to infer a property for the American option from the corresponding property for the European value.

Denote the payoff of the option by $h(s)=(s-K)^{+}$. Denote by $\left(S_{u}(t, s)\right)_{t \leq u \leq T}$ the stock price process given initial condition $S_{t}=s$, for $t \in[0, T]$. Recall that the American ESO value is given by

$$
V(t, s)=\sup _{\tau \in \mathcal{T}_{t, t}^{L}} E\left[e^{-r(\tau-t)} h\left(S_{\tau}(t, s)\right)\right], \quad 0 \leq t \leq T, \quad s>0 .
$$


For fixed $(t, s)$ the process $\left(e^{-r(u-t)} V\left(u, S_{u}(t, s)\right)\right)_{t \leq u \leq T}$ is the smallest supermartingale that dominates $\left(e^{-r(u-t)} h\left(S_{u}(t, s)\right)\right)_{t \leq u \leq T}$. We now construct $V$ by an iterative procedure found in [4], adapted to the situation in hand.

For any continuous Borel function $g:[0, T] \times \mathbb{R}^{+} \rightarrow \mathbb{R}$, we define

$$
\left(R_{u} g\right)(t, s):=E\left[e^{-r(u-t)} g\left(u, S_{u}(t, s)\right)\right], \quad 0 \leq t \leq u \leq T, \quad s>0 .
$$

So, in particular, we have $V^{E}(t, s ; u)=\left(R_{u} h\right)(t, s)$ and this is decreasing in $t$ for $r=0, s>K$ and $C \geq \log K$.

Define the operator

$$
(\mathcal{K} g)(t, s):=\sup _{u \in[t, T]}\left(R_{u} g\right)(t, s), \quad 0 \leq t \leq T, \quad s>0 .
$$

It is straightforward to see that $\mathcal{K} h$ also exhibits time decay. Moreover, for $r=0$ and $s>K$, $\mathcal{K} h \geq h$ if $C \geq \log K$, by virtue of the Jensen inequality, since we have for any $u \in[t, T]$ :

$$
\begin{aligned}
(\mathcal{K} h)(t, s) \geq\left(R_{u} h\right)(t, s) & =e^{-r(u-t)} E\left[\left(S_{u}(t, s)-K\right)^{+}\right] \\
& \geq e^{-r(u-t)}\left(E\left[S_{u}(t, s)\right]-K\right)^{+} \\
& =e^{-r(u-t)}\left(s \exp \left[\beta(t, \log s)(u-t)+\frac{1}{2} \Sigma^{2}(t ; u)\right]-K\right)^{+} .
\end{aligned}
$$

Then, if $r=0$, we see that $(\mathcal{K} h)(t, s) \geq h(s)$ provided that

$$
\beta(t, \log s)(u-t)+\frac{1}{2} \Sigma^{2}(t ; u) \geq 0 .
$$

This is (20), so for $s>K$ will hold whenever $C \geq \log K$.

Since $\mathcal{K} h \geq h$, we have $\mathcal{K}^{n+1} h \geq \mathcal{K}^{n} h$, where $\mathcal{K}^{n}$ denotes the $n$-fold iterate of $\mathcal{K}$. We can thus define

$$
w:=\lim _{n \rightarrow \infty} \mathcal{K}^{n} h=\sup _{n \in \mathbb{N}} \mathcal{K}^{n} h .
$$

It is easy to see that $w$ inherits the properties of $\mathcal{K} h$, so $w$ also exhibits time decay.

The remainder of the proof follows the same reasoning as Theorem 9.4 in El Karoui et al [4], to establish that $w$ is the smallest fixed point of $\mathcal{K}$ dominating $h$, and hence that $w$ coincides with $V$, so that $V$ also displays time decay when $r=0$ and $C \geq \log K$. Since $V$ displays time decay when $C \leq \log K$, we conclude that time decay holds for all values of $C$ when $r=0$. This ends the proof. For completeness, here is the argument.

We have $w \geq \mathcal{K}^{n+1} w=\mathcal{K}\left(\mathcal{K}^{n} w\right)$. Letting $n \rightarrow \infty$, we obtain $w \geq \mathcal{K} w$. The reverse inequality is trivial.

If $u$ is a fixed point of $\mathcal{K}$ dominating $h$, then $u=\mathcal{K}^{n} u \geq \mathcal{K}^{n} h$. Letting $n \rightarrow \infty$, we obtain $u \geq w$.

Fix $(t, s)$ and consider $Z_{u}=e^{-r(u-t)} w\left(u, S_{u}(t, s)\right)$. For $0 \leq t \leq u_{1} \leq u_{2} \leq T$, we have

$$
\begin{aligned}
E\left[Z_{u_{2}} \mid \mathcal{F}_{u_{1}}^{L}\right] & =e^{-r\left(u_{1}-t\right)} E\left[e^{-r\left(u_{2}-u_{1}\right)} w\left(u_{2}, S_{u_{2}}(t, s)\right) \mid \mathcal{F}_{u_{1}}^{L}\right] \\
& =e^{-r\left(u_{1}-t\right)}\left(R_{u_{2}} w\right)\left(u_{1}, S_{u_{1}}(t, s)\right) \\
& \leq e^{-r\left(u_{1}-t\right)}(\mathcal{K} w)\left(u_{1}, S_{u_{1}}(t, s)\right) \\
& =Z_{u_{1}} .
\end{aligned}
$$

Thus, $Z$ is a supermartingale dominating $e^{-r(u-t)} h\left(S_{u}(t, s)\right)$, and so must dominate $e^{-r(u-t)} V\left(u, S_{u}(t, s)\right)$ as well. In particular, $w(t, s)=Z_{0} \geq V(t, s)$.

For the reverse inequality, we observe from the supermartingale property for $e^{-r(u-t)} V\left(u, S_{u}(t, s)\right)$ that $\left(R_{u} V\right)(t, s) \leq V(t, s)$, and hence $\mathcal{K} V \leq V$. Therefore, $V$ is a fixed point of $\mathcal{K}$, and being a fixed point of $\mathcal{K}, V$ must dominate $w$. Hence, $V$ and $w$ coincide, and so $V$ inherits the properties of $w$, and we are done. 
Lemma 3. Suppose the map $t \rightarrow V(t, s)$ is non-increasing. Then the exercise boundary $s^{*}(t)$ is non-increasing, for $t \in[0, T]$.

Proof. Choose $(t, s) \in \mathcal{C}$ for some $s \in \mathbb{R}^{+}$and consider $t_{0}$ satisfying $0 \leq t_{0}<t \leq T$. By assumption, $V\left(t_{0}, s\right) \geq V(t, s)$, and therefore

$$
V\left(t_{0}, s\right)-(s-K)^{+} \geq V(t, s)-(s-K)^{+}>0, \quad 0 \leq t \leq T,
$$

so that $\left(t_{0}, s\right)$ is also in $\mathcal{C}$. That is, for $t_{0}<t$, we have that $s<s^{*}(t)$ necessarily implies that $s<s^{*}\left(t_{0}\right)$, and this can only be true if $s^{*}\left(t_{0}\right)$ is at least as big as $s^{*}(t)$, that is, $s^{*}\left(t_{0}\right) \geq s^{*}(t)$.

This lemma implies that $x^{*}(t)=\log s^{*}(t)$ is also non-increasing.

\subsection{Smooth fit condition}

In this subsection we establish the smooth-fit condition for $F$. There are three ingredients in the proof: convexity $F$ in $x$ (Theorem 1), a 'regularity' property of the exercise boundary $x^{*}$ (Lemma 4) and a result (Lemma 5) which allows us to establish a lower bound for $F_{x}$ just below the exercise boundary.

The smooth-fit condition for $F$ is as follows. The proof is given at the end of this subsection, after establishing some auxiliary lemmas.

Theorem 4. Suppose the exercise boundary is non-increasing. Then the value function satisfies the smooth fit condition, that is

$$
F_{x}\left(t, x^{*}(t)\right)=e^{-r t} e^{x^{*}(t)} \quad \Longleftrightarrow \quad V_{s}\left(t, s^{*}(t)\right)=1, \quad \text { for all } t \in[0, T) .
$$

When the exercise boundary is non-increasing, we have the regularity result below characterising the boundary. It states that if the log-stock price process starts arbitrarily close to the boundary, then it will hit the boundary in the next instant. This is in the spirit of the definition of a regular boundary point in the context of the Dirichlet problem (see Definition 4.2.9 and Theorem 4.2 .12 in [10]).

Lemma 4. Suppose the exercise boundary is non-increasing. Denote by $\tau^{*}(t, x)$ the optimal stopping time for $F(t, x)$, for some $(t, x) \in[0, T) \times \mathbb{R}$. Then, we have

$$
\lim _{\epsilon \rightarrow 0} \tau^{*}\left(t, x^{*}(t)-\epsilon\right) \rightarrow t, \quad \text { a.s., } \quad 0 \leq t \leq T .
$$

Proof. Without loss of generality, set the starting time to zero, write $X(x) \equiv X(0, x)$ for the value of the $\log$-stock price given $X_{0}=x$, as well as $\tau^{*}(x) \equiv \tau^{*}(0, x)$ and $x^{*}(0) \equiv x^{*}$. For $\epsilon>0$, since the exercise boundary is non-increasing we have

$$
\tau^{*}\left(x^{*}-\epsilon\right) \leq \inf \left\{\rho \in[0, T) \mid X_{\rho}\left(x^{*}-\epsilon\right) \geq x^{*}\right\} .
$$

From the solution (7) for $X(x)$ and the Law of the Iterated Logarithm for Brownian motion (Section I.16 of Rogers and Williams [17]), we have

$$
\sup _{0 \leq u \leq \rho} X_{u}(x)>x, \quad \text { a.s. }
$$

for every $\rho>0$. Hence there exists a sufficiently small $\epsilon>0$ such that

$$
\sup _{0 \leq u \leq \rho} X_{u}\left(x^{*}-\epsilon\right) \geq x^{*}, \quad \text { a.s. }
$$

Hence the right hand side of (21) tends to zero as $\epsilon \rightarrow 0$, and this completes the proof. 
The next ingredient we need for the proof of smooth fit is the following lemma.

Lemma 5. Let $(t, x) \in[0, T) \times \mathbb{R}$ and denote by $X(t, x)$ the log-stock price with initial condition $X_{t}=x$. Denote by $\left(\tau_{\epsilon}\right)_{\epsilon>0}$ a family of $\mathcal{T}_{t, T}^{L}$-stopping times converging to $t$ almost surely as $\epsilon \rightarrow 0$. Then $X(t, x)$ satisfies

$$
\lim _{\epsilon \rightarrow 0} \frac{1}{\epsilon}\left(\exp \left(X_{\tau_{\epsilon}}(t, x)\right)-\exp \left(X_{\tau_{\epsilon}}(t, x-\epsilon)\right)\right)=e^{x}, \quad \text { a.s. }
$$

Proof. Without loss of generality, consider a starting time $t=0$. The same ideas apply to any starting time $t \in[0, T)$. Write $X(x) \equiv X(0, x)$ for the log-stock price with initial condition $X_{0}=x \in \mathbb{R}$. For $\epsilon>0$, define

$$
\Delta_{u}(\epsilon):=\frac{1}{\epsilon}\left(\beta\left(u, X_{u}(x)\right)-\beta\left(u, X_{u}(x-\epsilon)\right)\right), \quad 0 \leq u<T .
$$

Using (12), we have

$$
\frac{1}{\epsilon}\left(e^{X_{\tau_{\epsilon}}(x)}-e^{X_{\tau_{\epsilon}}(x-\epsilon)}\right)=\frac{1}{\epsilon} e^{X_{\tau_{\epsilon}}(x-\epsilon)}\left[\exp \left\{\epsilon\left(1+\int_{0}^{\tau_{\epsilon}} \Delta_{u}(\epsilon) d u\right)\right\}-1\right] .
$$

Using Taylor's expansion, we get

$$
\frac{1}{\epsilon}\left(e^{X_{\tau_{\epsilon}}(x)}-e^{X_{\tau_{\epsilon}}(x-\epsilon)}\right)=e^{X_{\tau_{\epsilon}}(x-\epsilon)}\left(1+\int_{0}^{\tau_{\epsilon}} \Delta_{u}(\epsilon) d u+O(\epsilon)\right),
$$

where $O(\epsilon)$ denotes terms of order $\epsilon$ or higher. Observe that

$$
\lim _{\epsilon \rightarrow 0} \Delta_{u}(\epsilon)=b\left(u, X_{u}(x)\right) D_{u}(x), \quad 0 \leq u \leq T, \quad \text { a.s. },
$$

where $b, D$ are defined in (13). Then, using the fact that $\lim _{\epsilon \rightarrow 0} \tau_{\epsilon}=0$ (since we have set $t=0$ ) and $e^{X_{\tau_{\epsilon}}(x-\epsilon)}=e^{x}$ a.s. completes the proof.

Note that if the drift $\beta$ was constant or a deterministic function of time, then the lemma would follow directly from the fact that $\Delta$ in (22) is equal to zero.

We now prove the smooth fit condition.

Proof of Theorem 4 (Smooth fit). It entails no loss of generality if we set $r=0$ and $t=0$, but significantly simplifies notation. Write $F(x) \equiv F(0, x)$ and $x^{*} \equiv x^{*}(0)$. Then $F(x)=\left(e^{x}-K\right)$ for $x \geq x^{*}$, so $F_{x}\left(x^{*}+\right)=e^{x^{*}}$.

On the other hand, from Theorem 1 we know that the mapping $x \rightarrow F(x)$ is increasing and convex, so $F_{x}\left(x^{*}-\right) \leq e^{x^{*}}$. Hence it suffices to show that

$$
F_{x}\left(x^{*}-\right) \geq e^{x^{*}} .
$$

As before let $\tau^{*}(x)$ denote the optimal stopping time for starting state $x$. Since $\tau^{*}(x-\epsilon)$ is suboptimal for $F(x)$, we have

$$
\begin{aligned}
F(x)-F(x-\epsilon) & \geq E\left[\left(\exp \left(X_{\tau^{*}(x-\epsilon)}(x)\right)-K\right)^{+}-\left(\exp \left(X_{\tau^{*}(x-\epsilon)}(x-\epsilon)\right)-K\right)^{+}\right] \\
& \geq E\left[\left(e^{X_{\tau^{*}(x-\epsilon)}(x)}-e^{X_{\tau^{*}(x-\epsilon)}(x-\epsilon)}\right) \mathbb{1}_{\left\{X_{\tau^{*}(x-\epsilon)}(x-\epsilon) \geq \log K\right\}}\right],
\end{aligned}
$$

where we have use the fact that $x \rightarrow X(x)$ is increasing.

By Lemma 4 and the fact that it is never optimal to exercise below the strike, we have

$$
\lim _{\epsilon \rightarrow 0} \mathbb{1}_{\left\{X_{\tau\left(x^{*}-\epsilon\right)}\left(x^{*}-\epsilon\right) \geq \log K\right\}}=\mathbb{1}_{\left\{X_{0}\left(x^{*}\right) \geq \log K\right\}}=1, \quad \text { a.s. }
$$

Also, by Lemma 5 , we have

$$
\lim _{\epsilon \rightarrow 0} \frac{1}{\epsilon}\left(e^{X_{\tau\left(x^{*}-\epsilon\right)}\left(x^{*}\right)}-e^{X_{\tau\left(x^{*}-\epsilon\right)}\left(x^{*}-\epsilon\right)}\right)=e^{x^{*}}, \quad \text { a.s. }
$$


Using (24) and (25) and noting that all terms inside the expectation in (23) are uniformly integrable, Theorem II.21.2 in Rogers \& Williams [17] gives

$$
\lim _{\epsilon \rightarrow 0} \frac{1}{\epsilon}\left[F\left(x^{*}\right)-F\left(x^{*}-\epsilon\right)\right] \geq e^{x^{*}},
$$

which completes the proof.

\subsection{The early exercise decomposition}

We now transform the state space from log-stock price to stock price in order to state the early exercise decomposition for the ESO value function $V$, given by $e^{-r t} V(t, s)=F(t, \log s)$. With this change of variable the smooth fit condition becomes $V_{s}\left(t, s^{*}(t)\right)=1$ and the PDE for $F$ in the continuation region transforms to $\mathcal{L}^{S} V-r V=0$, where $\mathcal{L}^{S}$ is the extended generator of $S$, given by

$$
\mathcal{L}^{S}=\frac{\partial}{\partial t}+\alpha(t, s) s \frac{\partial}{\partial s}+\frac{1}{2} \sigma^{2} s^{2} \frac{\partial^{2}}{\partial s^{2}}, \quad \alpha(t, s)=\beta(t, \log s)+\frac{1}{2} \sigma^{2} .
$$

We then have the following decomposition for $V$.

Theorem 5. The value function $V$ of an executive stock option with strike $K$ and maturity $T$ has the following decomposition into a European option value and an early exercise premium:

$$
V(t, s)=e^{-r(T-t)} E\left[\left(S_{T}-K\right)^{+} \mid S_{t}=s\right]+p(t, s), \quad(t, s) \in[0, T] \times \mathbb{R}^{+},
$$

where

$$
p(t, s):=\int_{t}^{T} e^{-r(u-t)} E\left[\left(\left(r-\alpha\left(u, S_{u}\right)\right) S_{u}-r K\right) \mathbb{1}_{\left\{S_{u}>s^{*}(u)\right\}} \mid S_{t}=s\right] d u,
$$

is the early exercise premium.

Proof. The smooth fit condition implies that $F_{x}$ is continuous. We have that $F_{x x}$ is continuous in the continuation region and equal to $e^{-r t+x}$ in the stopping region. Though the second derivative might not be continuous across the exercise boundary we may nevertheless apply the generalised Itô formula for convex functions to $F$, to obtain the Doob-Meyer decomposition of the Snell envelope:

$$
\begin{gathered}
F\left(T, X_{T}\right)=F\left(t, X_{t}\right)+\sigma \int_{t}^{T} F_{x}\left(u, X_{u}\right) d B_{u}^{L} \\
+\int_{t}^{T} e^{-r u}\left[\left(\beta\left(u, X_{u}\right)+\frac{1}{2} \sigma^{2}-r\right) e^{X_{u}}+r K\right] \mathbb{1}_{\left\{X_{u}>x^{*}(u)\right\}} d u, \quad 0 \leq t \leq T,
\end{gathered}
$$

where we have used $F(t, x)=e^{-r t}\left(e^{x}-K\right)$ for $x>x^{*}(t)$. Now take expectation conditional on $X_{t}=x$ (and of course given a known value of $L$ ), change variables from $X$ to $S$, and (28) follows.

\subsection{Integral equation for early exercise boundary}

The integral equation for the early exercise boundary follows by setting $s=s^{*}(t)$ in (28), yielding the following corollary. To be explicit we restore the dependence on the random variable $L$. For $L=\ell$, denote the insider's exercise boundary by $s_{\ell}^{*}(t)$. Using $V\left(t, s_{\ell}^{*}(t)\right)=s_{\ell}^{*}(t)-K$, we obtain:

Corollary 1. For $L=\ell \in \mathbb{R}$, the insider's exercise boundary $s_{\ell}^{*}$ satisfies, for $0 \leq t \leq T$,

$$
\begin{aligned}
& s_{\ell}^{*}(t)=K+e^{-r(T-t)} E\left[\left(S_{T}-K\right)^{+} \mid S_{t}=s^{*}(t), L=\ell\right] \\
+ & \int_{t}^{T} e^{-r(u-t)} E\left[\mathbb{1}_{\left\{S_{u}>s_{\ell}^{*}(u)\right\}}\left(\left(r-\alpha\left(u, S_{u}\right)\right) S_{u}-r K\right) \mid S_{t}=s_{\ell}^{*}(t), L=\ell\right] d u .
\end{aligned}
$$




\subsection{Numerical solution of early exercise boundary equation}

The algorithm used to numerically solve the integral equation in Corollary 1 is as follows. For a fixed $t \in[0, T]$ and $\ell \in \mathbb{R}$, we treat the computations of the expectations as European option prices, with stock price dynamics under $\mathbb{F}^{L}$ given by

$$
d S_{u}=\alpha\left(u, S_{u}\right) S_{u} d u+\sigma S_{u} d B_{u}^{L}
$$

with $\alpha$ defined in (26).

These are computed by solving the associated Black-Scholes style PDE using a central difference scheme, for a sufficiently wide range of $s_{\ell}^{*}(t)$. We discretise the interval $[0, T]$ and use the trapezoidal rule to approximate the integral, solving the discretised integral equation using the fixed point method. The exercise boundary is computed by backward recursion with the starting value $s_{\ell}^{*}(T)=K$.

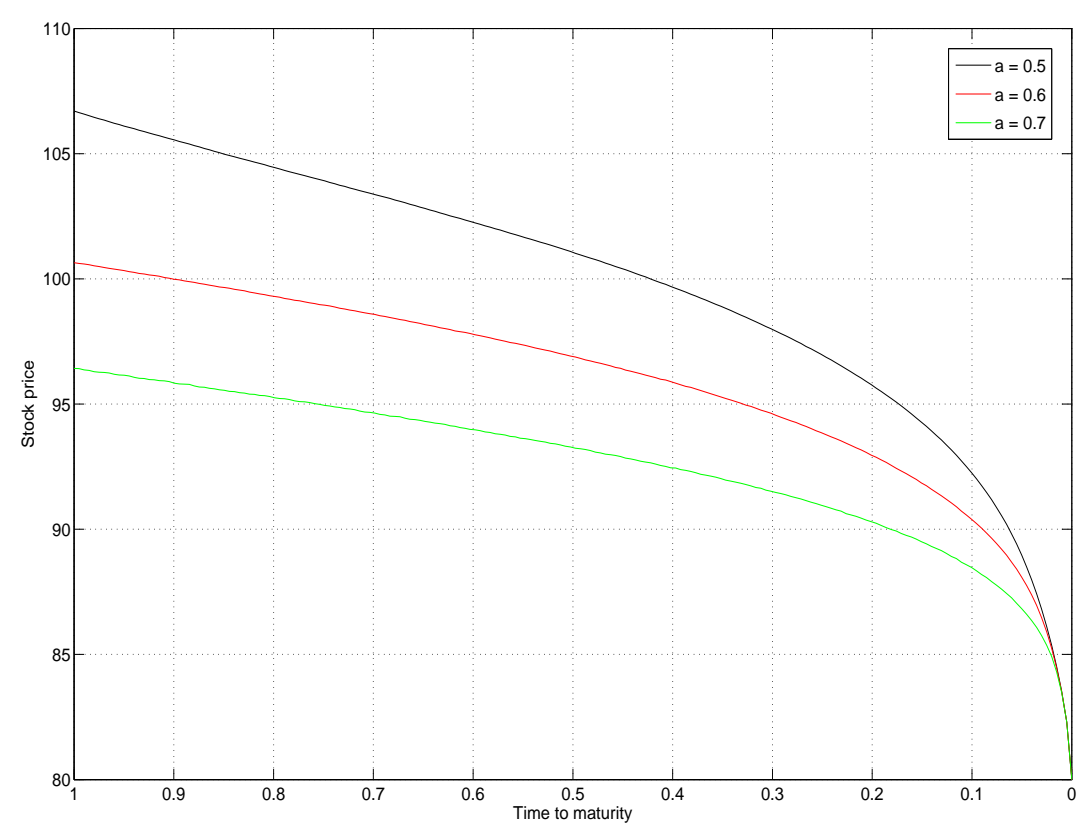

Figure 1: Insider's exercise boundaries for $L=a \log 80$ with different values of the noise coefficient $a, r=0.01, \mu=0.02, \sigma=0.2, T=1, K=80$.

Figure 1 shows the insider's exercise boundaries when the stock appreciation rate $\mu$ is higher than the interest rate. The insider possesses noisy log-stock price knowledge with $L=a \log 80$ with $a=0.5,0.6,0.7$, so the insider knows that the ESO is likely to be at-the-money at maturity with varying degrees of certainty.

The impact of inside information in this case is clear. Recall that it is not optimal for the regular agent to exercise early when $\mu \geq r .^{3}$ This conclusion is altered for the insider, who has greater certainty than the regular agent that the option will expire out of the money, and this induces early exercise. We also observe that the exercise boundary is lower as $a$ increases towards 1 , and the privileged information becomes less noisy. The insider becomes more certain that the option will expire worthless and early exercise is induced at lower thresholds.

Figure 2 shows the regular trader's and insider's exercise boundaries when $\mu<r$. The insider possesses noisy log-stock price knowledge where $L=0.5 \log S_{T}$ with $S_{T}=78,80,82,90$.

\footnotetext{
${ }^{3}$ Indeed, attempts to solve for the regular agent's exercise boundary numerically when $\mu \geq r$ leads to divergence when executing the fixed point method.
} 


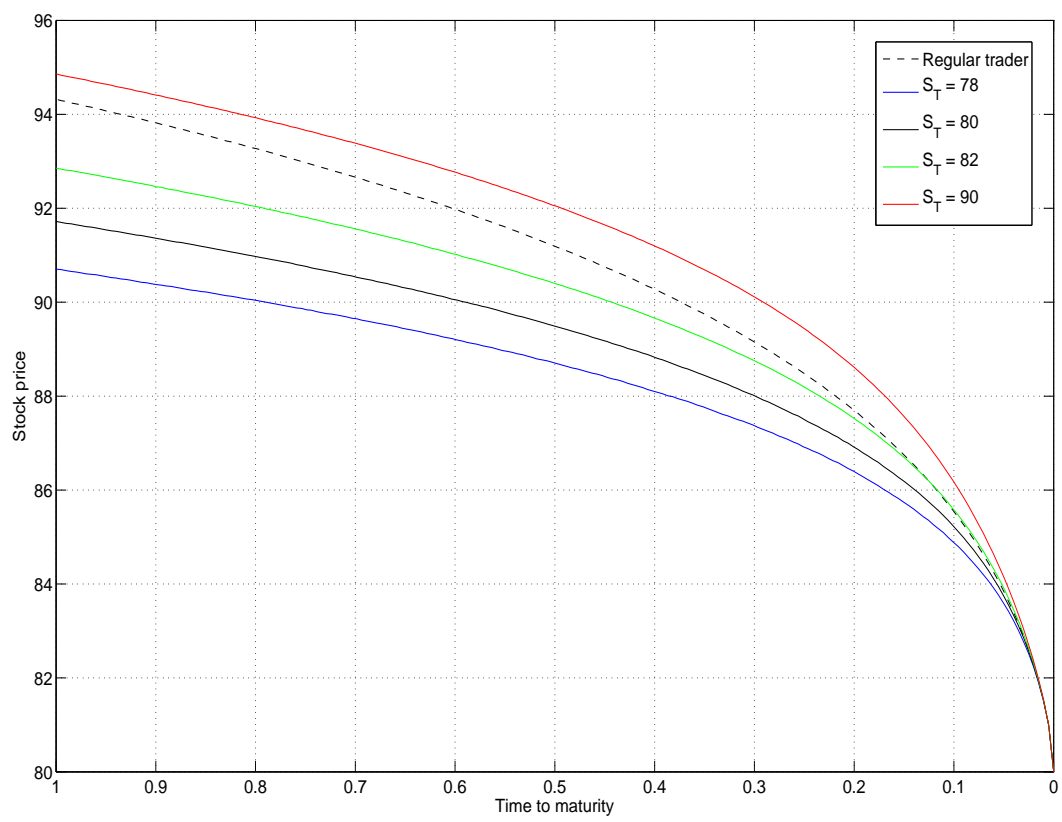

Figure 2: Regular trader's and insider's exercise boundaries for $L=0.5 \log S_{T}$ with different values of $S_{T}, r=0.01, \mu=0, \sigma=0.2, T=1, K=80$.

For $S_{T}=78$ and $S_{T}=80$, the insider has a lower exercise boundary than the regular agent due to his pessimistic inside information, in a similar vein to the results in Figure 1. For $S_{T}=82$, the insider knows that the ESO is likely to be in-the-money, yet still exercises the ESO earlier than the regular agent. Although the fact that the ESO is likely to end up in-the-money tends to delay exercise, there is a competing effect of a lower variance in the stock price as perceived by the insider, and this induces earlier exercise. For the case $S_{T}=90$, the privileged information is sufficiently optimistic so that the insider exercises later than the regular trader.

This suggests that inside information has two potentially competing effects: a reduced variance of the stock price that hastens exercise and a directional effect, which can hasten or delay exercise.

\section{Conclusions}

Using an initial enlargement of filtration to augment a Brownian filtration with noisy information on the value of a stock at the maturity time of an ESO, we have analysed the stopping decision faced by an insider who does not trade the stock or other securities. This shows that the insider can exercise the ESO before maturity, in situations in which a regular agent would not. This involved establishing fundamental properties of the value function (notably convexity, time decay and smooth fit) when the price process is a time-inhomogeneous diffusion.

This paper has set a framework in which such questions can be studied. An interesting direction for future work is to add trading opportunities in outside assets and risk aversion for the agent.

\section{References}

[1] A. Danilova, M. Monoyios, And A. NG, Optimal investment with inside information and parameter uncertainty, Math. Financ. Econ., 3 (2010), pp. 13-38. 
[2] E. Ekström, Properties of American option prices, Stochastic Process. Appl., 114 (2004), pp. 265278.

[3] N. El KAROUI, Les aspects probabilistes du contrôle stochastique, in Ninth Saint Flour Probability Summer School-1979 (Saint Flour, 1979), vol. 876 of Lecture Notes in Math., Springer, Berlin, 1981, pp. 73-238.

[4] N. El Karoui, M. Jeanblanc-Picqué, And S. E. Shreve, Robustness of the Black and Scholes formula, Math. Finance, 8 (1998), pp. 93-126.

[5] N. El Karoui, J.-P. Lepeltier, And A. Millet, A probabilistic approach to the reduite in optimal stopping, Probab. Math. Statist., 13 (1992), pp. 97-121.

[6] M. Grasselli And V. Henderson, Risk aversion and block exercise of executive stock options, J. Econom. Dynam. Control, 33 (2009), pp. 109-127.

[7] D. G. Hobson, Volatility misspecification, option pricing and superreplication via coupling, Ann. Appl. Probab., 8 (1998), pp. 193-205.

[8] S. D. JACKA AND J. R. LyNN, Finite-horizon optimal stopping obstacle problems and the shape of the continuation region, Stochastics Stochastics Rep., 39 (1992), pp. 25-42.

[9] M. Jeanblanc, M. Yor, And M. Chesney, Mathematical methods for financial markets, Springer Finance, Springer-Verlag London Ltd., London, 2009.

[10] I. Karatzas And S. E. Shreve, Brownian motion and stochastic calculus, vol. 113 of Graduate Texts in Mathematics, Springer-Verlag, New York, second ed., 1991.

[11] _ Methods of mathematical finance, vol. 39 of Applications of Mathematics (New York), Springer-Verlag, New York, 1998.

[12] T. Leung AND R. SiRCAR, Accounting for risk aversion, vesting, job termination risk and multiple exercises in valuation of employee stock options, Math. Finance, 19 (2009), pp. 99-128.

[13] _ Exponential hedging with optimal stopping and application to employee stock option valuation, SIAM J. Control Optim., 48 (2009), pp. 1422-1451.

[14] R. C. Merton, Theory of rational option pricing, Bell J. Econom. and Management Sci., 4 (1973), pp. 141-183.

[15] K. Muthuraman, A moving boundary approach to American option pricing, J. Econom. Dynam. Control, 32 (2008), pp. 3520-3537.

[16] L. C. G. Rogers and J. Scheinkman, Optimal exercise of executive stock options, Finance Stoch., 11 (2007), pp. 357-372.

[17] L. C. G. Rogers and D. Williams, Diffusions, Markov processes, and martingales. Vol. 1, Cambridge Mathematical Library, Cambridge University Press, Cambridge, 2000. Foundations, Reprint of the second (1994) edition.

[18] R. Sircar And W. Xiong, A general framework for evaluating executive stock options, J. Econom. Dynam. Control, 31 (2007), pp. 2317-2349.

[19] M. YoR, Some aspects of Brownian motion. Part II, Lectures in Mathematics ETH Zürich, Birkhäuser Verlag, Basel, 1997. Some recent martingale problems. 